\title{
Association of TCF7L2 rs7903146 Gene Polymorphism with the Risk of NAFLD and CAD in the Chinese Han Population
}

\author{
Xin Yan ${ }^{1,4}$, Wenwen Jin ${ }^{1}$, Jie Zhang ${ }^{1}$, Mengke Wang ${ }^{1}$, Shousheng Liu* ${ }^{2,3}$ and Yongning Xin*1,2,3 \\ ${ }^{1}$ Department of Infectious Disease, Qingdao Municipal Hospital, Qingdao, Shandong, China; ${ }^{2}$ Clinical Research Center, Qingdao \\ Municipal Hospital, Qingdao, Shandong, China; ${ }^{3}$ Digestive Disease Key Laboratory of Qingdao, Qingdao, Shandong, China; \\ ${ }^{4}$ Dalian Medical University, Dalian, Liaoning, China
}

\begin{abstract}
Background and Aims: Coronary artery disease (CAD) is a major cause of morbidity and mortality in patients with nonalcoholic fatty liver disease (NAFLD). Previous studies have suggested that TCF7L2 rs7903146 was related to the risk of developing NAFLD but the conclusions are not consistent and no related study has been conducted in Chinese populations. The aim of this study was to investigate the association between TCF7L2 rs7903146 and the risk of developing NAFLD and CAD in a Chinese Han population. Methods: TCF7L2 rs7903146 genotypes were measured by the MALDI-TOFMS from 143 NAFLD patients, 159 CAD patients, 131 NAFLD + CAD patients, and 212 healthy controls. The demographic data and serum lipid profiles of all subjects were collected. The distributions of genotype and allele frequency in each group were also tested. Logistic regression was used to investigate the risk of TCF7L2 rs7903146 with NAFLD and CAD. All statistical analyses were conducted using SPSS 23.0. Results: There were no significant differences in the distributions of TCF7L2 rs7903146 genotype and allele frequency in each of the two groups, and the TCF7L2 rs7903146 CT + TT genotype did not increase the risk of developing NAFLD, CAD, and NAFLD + CAD. Except for body mass index in the control group, the differences of clinical parameters between the TCF7L2 rs7903146 T allele carriers and non-carriers in each group were not significant. In the non-obese group, the TCF7L2 rs7903146 CT + TT genotype was a protective factor for the development of NAFLD in the non-obese subjects (odds ratio $=0.359,95 \%$ confidence interval: $0.134-0.961$, $p=0.041$ ). Conclusions: TCF7L2 rs7903146 was not associated with the risk of developing NAFLD, CAD, and NAFLD + CAD in the Chinese Han population. In the nonobese population, the TCF7L2 rs7903146 CT + TT genotype was a protective factor against the development of NAFLD.
\end{abstract}

Keywords: Nonalcoholic fatty liver disease; Coronary artery disease; TCF7L2; Single nucleotide polymorphism.

Abbreviations: ALP, alkaline phosphatase; BMI, body mass index; $C A D$, coronary artery disease; FPG, fasting plasma glucose; GGT, gamma-glutamyltransferase; GIP, glucose-dependent insulinotropic polypeptide; HDL, high-density lipoprotein; LDL, low-density lipoprotein; NAFLD, non-alcoholic fatty liver disease; SD, standard deviation; TBIL, total bilirubin; TC, total cholesterol; TCF-4, transcription factor-4; TG, triglyceride.

Received: 26 July 2020; Revised: 25 August 2020; Accepted: 24 September 2020 *Correspondence to: Yongning Xin, Department of Infectious Disease, Qingdao Municipal Hospital, 1 Jiaozhou Road, Qingdao, Shandong 266011, China. Tel: +86-532-82789463, Fax: +86-532-85968434, E-mail: xinyongning@163.com; Shousheng Liu, Clinical Research Center, Qingdao Municipal Hospital, 1 Jiaozhou Road, Qingdao, Shandong 266011, China. Tel: +86-532-88905831, Fax: +86532-88905293, E-mail: shoushengliuouc@163.com
Citation of this article: Yan X, Jin W, Zhang J, Wang M, Liu S, Xin Y. Association of TCF7L2 rs7903146 gene polymorphism with the risk of NAFLD and CAD in the Chinese Han population. J Clin Transl Hepatol 2020;8(4):371-376. doi: 10.14218/JCTH.2020.00071.

\section{Introduction}

Non-alcoholic fatty liver disease (NAFLD) is the clinicopathological syndrome, characterized by excessive intracellular fat deposition that occurs with the exclusion of alcohol and other specific liver damaging factors, such as viruses, drugs, autoimmune factors, and genetic factors. ${ }^{1}$ According to the clinical statistic results, coronary artery disease (CAD) causes the highest mortality in the patients with NAFLD, and the incidence of CAD in patients with NAFLD has increased significantly in recent years. ${ }^{2,3}$ A previous study showed that NAFLD can act as one of the independent risk factors for $C A D$, after excluding hypertension, obesity, diabetes, and other factors. ${ }^{4}$ Both NAFLD and CAD are influenced by the interaction of genetic and environmental factors, and they share some pathological mechanisms, such as obesity, insulin resistance, dyslipidemia, inflammation, and oxidative stress. ${ }^{3,5,6}$ A large number of studies have investigates the role of NAFLD in the development of CAD. NAFLD was reported to be related to the formation of coronary artery plaques and impaired coronary blood flow reserve. ${ }^{4,7,8}$ In addition, genetic pathogenesis in NAFLD and CAD is drawing more attention, and the gene polymorphisms of $A D I P O Q$, LEPR, APOC3, PPAR, SREBP, TM6SF2, and MTTP have been reported as risk factors for the development of NAFLD and CAD. ${ }^{5}$

TCF7L2 is located on chromosome $10 \mathrm{q} 25$ and encodes the transcription factor-4 (TCF-4) (also known as lymphocyte factor-4), which is an important member of the transcription factor family. ${ }^{9-11}$ The TCF-4 family can participate in a variety of physiological pathways in different types of cells. ${ }^{12,13}$ In 2009, Musso et al. ${ }^{14}$ first reported that TCF7L2 rs7903146 T allele frequency was higher in NAFLD patients than in healthy controls. In addition, they found that TCF7L2 rs7903146 T allele carriers possessed higher levels of low-density lipoprotein (LDL), very low-density lipoprotein, triglyceride (TG), and cytokeratin 18 than non-carriers in the NASH subgroup. Subsequently, Giovanni et al. ${ }^{15}$ reported that TCF7L2 rs7903146 T allele carriers had the prolonged elevation of post-meal glucose-dependent insulinotropic polypeptide (GIP). The elevated GIP was demonstrated in other studies 
Yan X. et al: Association of TCF7L2 with NAFLD and CAD

to exacerbate liver steatosis and increase the levels of postprandial resistin and free fatty acids. ${ }^{16,17}$ Besides, some evidence has suggested that TCF7L2 rs7903146 is associated with serum lipid indexes, including TG, total cholesterol (TC), LDL, and apolipoprotein B, all of which are associated with risk of CAD development. ${ }^{18,19}$ Corella et al. ${ }^{20}$ demonstrated that the relationship between TCF7L2 rs7903146 and serum lipid profiles was influenced by adherence to the MedDiet. When adherence was low, the levels of TC and LDL were higher in $T$ carriers than $\mathrm{CT} / \mathrm{CC}$ carriers. But when adherence was high, no difference was observed between TT carriers and CT/CC carriers. Up to now, the association of TCF7L2 rs7903146 with the risk of developing NAFLD and CAD was investigated only in a limited amount of studies, and no related report was available in Chinese populations. In addition, the effect of TCF7L2 rs7903146 polymorphism on serum lipid profiles is still unclear. ${ }^{21-23}$ In consideration of the increased incidence of NAFLD and CAD in China, illuminating the association of TCF7L2 rs7903146 with the risk of NAFLD and $C A D$ in Chinese may provide some new ideas for the treatment of NAFLD and CAD.

The aim of this study was to investigate the relationship of TCF7L2 rs7903146 with the risk of developing NAFLD and $C A D$ in a Chinese Han population, and explore the effect of TCF7L2 rs7903146 on the serum lipid profiles.

\section{Methods}

\section{Subjects}

This study was conducted according to the principles of the declaration of Helsinki and its appendices. ${ }^{24}$ Subjects were composed of 143 patients with NAFLD, 159 patients with CAD, 131 patients with NAFLD + CAD, and 212 healthy controls, who were enrolled at the Qingdao Municipal Hospital (China) from December 2017 to December 2018. The Guidelines for the Diagnosis and Treatment of Non-alcoholic Fatty Liver Disease (2010 Revision) and the Guidelines for the Diagnosis and Treatment of Acute ST-Segment Elevation Myocardial Infarction (2015 Version) were used as exclusion and inclusion criteria for NAFLD and CAD. Patients with NAFLD were included from the Department of Gastroenterology, in a random manner, and diagnosed by B-type ultrasonography. Combining the medical history and laboratory testing, subjects with alcoholic fatty liver, viral hepatitis, autoimmune liver disease, drug-induced liver disease and hereditary metabolic disease were excluded. Patients with CAD were included from the Department of Cardiology, in a random manner, and diagnosed by coronary angiography. We divided the CAD patients into with or without NAFLD groups, respectively. The healthy controls were included from the Physical Examination Center of the Qingdao Municipal Hospital, in a random manner. All the subjects signed informed consent forms after participating in this study.

\section{Biochemical analyses}

The demographic data (gender, age, height and weight) were obtained by questionnaire. The body mass index (BMI) was calculated by weight $(\mathrm{kg}) /$ height $(\mathrm{m})^{2}$. Fasting venous blood samples of each subject were collected after a 12-h overnight fasting and kept in ethylene diamine tetraacetic acid-containing tubes. The biochemical data, such as TG, TC, fasting plasma glucose (FPG), high-density lipoprotein (HDL), LDL, alkaline phosphatase (ALP), gamma-glutamyltransferase (GGT), and total bilirubin (TBIL) were measured through standard laboratory method.

\section{Genomic DNA extraction and genotyping}

Genomic DNA was extracted from each blood sample and stored at $-80^{\circ} \mathrm{C}$ until use. The primers for PCR amplification were designed and synthesized by Bomiao Biotechnology (Beijing, China) as: 5'-ACGTTGGATGAACTAAGGGTGCCTCATACG-3' and 5'-ACGTTGGATGGCCTCAAAACCTAGCACAGC-3'. The detailed process of PCR amplification consists of the following steps: an initial denaturation at $94^{\circ} \mathrm{C}$ for $5 \mathrm{~m}$, followed by 45 cycles of denaturing at $94^{\circ} \mathrm{C}$ for $20 \mathrm{~s}$, annealing at $56^{\circ} \mathrm{C}$ for $30 \mathrm{~s}$, and extending at $72^{\circ} \mathrm{C}$ for $1 \mathrm{~m}$. After that, a final extension at $72{ }^{\circ} \mathrm{C}$ was conducted for $5 \mathrm{~m}$. The genotype TCF7L2 rs7903146 was detected by DNA sequence using the MALDI-TOF-MS (MassARRAY System; Agena Bioscience, Shanghai, China) and raw data was acquired by TYPER4.0.

\section{Statistical analysis}

The measurement data were expressed as mean \pm standard deviation (SD) for those conforming to the normal distribution, otherwise as median quartile. Homogeneity of variance was tested between each of two groups. The independent samples $t$-test was used for data conforming to normal distribution with homogeneity variances, while the rest were tested by rank sum test. Chi-square test was used to analyze the gender distribution differences. Hardy-Weinberg equilibrium was performed by chi-square test. The distribution of genotypes and allele frequency between two groups were also tested by chisquare. The correlation between genotype and risk of diseases were estimated by binary logistic regression modeling. The demographic and biochemical data of different genotypes were tested by independent sample $t$-test and rank sum test. A $p$-value of $<0.05$ was considered to indicate statistical significance. All the statistical analyses were conducted by SPSS 23.0 software (IBM Corp., Armonk, NY, USA).

\section{Results}

\section{Demographic and biochemical characteristics of subjects}

A total of 457 subjects were included in this study. As the results show in Table 1, differences of gender distribution were observed between the NAFLD group and control group, and between the NAFLD + CAD group and the control group. The NAFLD group had higher levels of BMI, TC, TG, LDL, alanine aminotransferase, GGT and FPG than the control group, while age and HDL level were lower in the NAFLD group compared to the control group (all $p<0.05$ ). No significant differences were observed for aspartate aminotransferase, ALP and TBIL between the NAFLD group and the control group. Age, BMI and levels of TG, alanine aminotransferase, ALP, GGT, and FPG in the CAD group were high compared to those in the control group, and the levels of TC, HDL, and LDL were low in the CAD group compared to those in the control group (all $p<0.05$ ). There were higher age, BMI, and levels of TG, alanine aminotransferase, ALP, GGT and FPG, and lower levels of TC, HDL and LDL in the NAFLD + CAD group compared to the control group (all $p<0.05$ ). The NAFLD + CAD group had higher age and levels of ALP and FPG, and lower BMI and levels of $\mathrm{HDL}, \mathrm{LDL}$, alanine aminotransferase and TC than the NAFLD group (all $p<0.05$ ). 


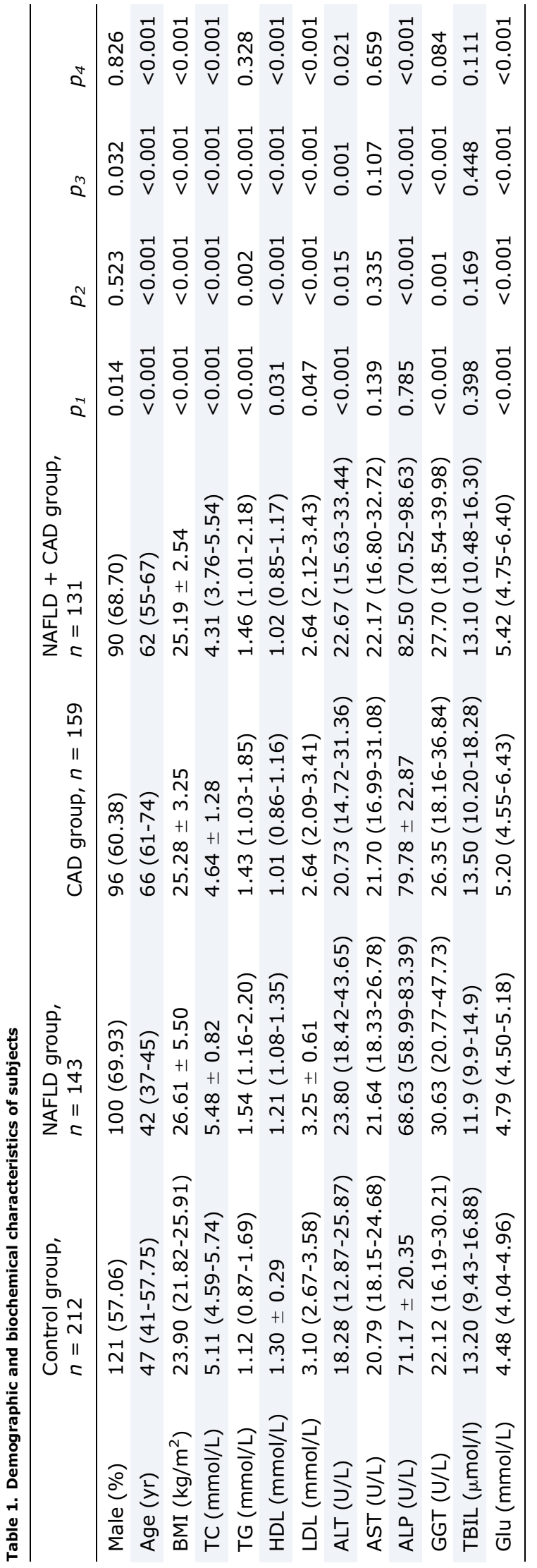

TCF7L2 rs7903146 genotype and allele frequency distribution

The genotype frequency distribution of TCF7L2 rs7903146 was in accordance with Hardy-Weinberg equilibrium in each group (all $p>0.05$ ) (Table 2). As the results show in Table 3, there was no significant difference in the genotype distribution of TCF7L2 rs7903146 (CC vs. CT + TT) between the NAFLD group and the control group, the CAD group and the control group, the NAFLD + CAD group and the control group, and the NAFLD + CAD group and the NAFLD group (all $p>$ 0.05). In addition, the difference of TCF7L2 rs7903146 allele frequency distribution in each group was also not significant (all $p>0.05$ ). When we analyzed the association of TCF7L2 rs7903146 genotype and the risk of NAFLD, CAD, and NAFLD $+C A D$, the results suggested that the $C T+T T$ genotype did not increase the risk of developing NAFLD, CAD, and NAFLD + $C A D$ in the general population, and the $C T+\Pi$ genotype also did not increase the risk of developing CAD in the patients with NAFLD (Table 4).

\section{Association of TCF7L2 rs7903146 genotype with the risk of NAFLD in the non-obese subjects}

The difference of clinical parameters between the $T$ allele carriers and non-carriers in each group was analyzed, and no significant difference was observed in the NAFLD group, the CAD group, and the NAFLD + CAD group (data not shown). However, the BMI of the T allele carriers was lower than in non-carriers in the control group $(p=0.027)$. To investigate the potential relationship of TCF7FL2 rs7903146 genotype with the BMI value, we divided the whole subject population into two groups: obese subjects (BMI $\geq 25$ ) and non-obese subjects (BMI <25). In the obese group, no significant difference was observed for the genotype distribution of TCF7L2 rs7903146 (CC vs. CT + TT) between the NAFLD patients and non-NAFLD patients. In the non-obese group, distribution of the $\mathrm{CT}+\pi$ genotype and CC genotype was significantly different $(p=0.035)$ (Table 5$)$. Logistic regression model analysis suggested that the CT + TT genotype was a protective factor against the development of NAFLD in the nonobese subjects (odds ratio $=0.359,95 \%$ confidence interval: $0.134-0.961, p=0.041$ ). After adjustment for gender and age, and serum lipids, the CT + TT genotype was still a protective factor against the development of NAFLD in the nonobese subjects (odds ratio $=0.245,95 \%$ confidence interval: $0.072-0.837, p=0.025$ ) (Table 6).

Table 2. Result of Hardy-Weinberg Equilibrium analysis

\begin{tabular}{llll}
\hline Gene locus & Group & $X^{2}$ & $p$ \\
\hline rs7903146 & NAFLD group & 0.39 & 0.53 \\
& Control group & 0.63 & 0.43 \\
& CAD group & 0.03 & 0.86 \\
& NALFD + CAD group & 0.07 & 0.81 \\
\hline
\end{tabular}

${ }^{*} p<0.05$ was considered different.

Abbreviations: CAD, coronary artery disease; NAFLD, nonalcoholic fatty liver disease. 


\begin{tabular}{|c|c|c|c|c|c|c|c|c|c|c|c|c|}
\hline & \multicolumn{2}{|c|}{ Genotype frequency, $n(\%)$} & \multirow[b]{2}{*}{$p_{1}$} & \multirow[b]{2}{*}{$p_{2}$} & \multirow[b]{2}{*}{$p_{3}$} & \multirow[b]{2}{*}{$p_{4}$} & \multicolumn{2}{|c|}{$\begin{array}{l}\text { Allele frequency, } \\
n(\%)\end{array}$} & \multirow[b]{2}{*}{$p_{1}$} & \multirow[b]{2}{*}{$p_{2}$} & \multirow[b]{2}{*}{$p_{3}$} & \multirow[b]{2}{*}{$p_{4}$} \\
\hline & $\mathrm{CC}$ & $\mathrm{CT}+\mathrm{TT}$ & & & & & $\mathrm{C}$ & $\mathrm{T}$ & & & & \\
\hline NAFLD & $125(89.93)$ & $14(10.07)$ & 0.93 & 0.28 & 0.06 & 0.08 & $\begin{array}{l}263 \\
(94.60)\end{array}$ & $\begin{array}{l}15 \\
(5.40)\end{array}$ & 0.90 & 0.22 & 0.06 & 0.06 \\
\hline Control & $190(89.62)$ & $22(10.38)$ & & & & & $\begin{array}{l}402 \\
(94.81)\end{array}$ & $\begin{array}{l}22 \\
(5.19)\end{array}$ & & & & \\
\hline CAD & $134(85.90)$ & $22(14.10)$ & & & & & $\begin{array}{l}289 \\
(92.63)\end{array}$ & $\begin{array}{l}23 \\
(7.37)\end{array}$ & & & & \\
\hline $\begin{array}{l}\text { NAFLD + } \\
\text { CAD }\end{array}$ & $110(95.65)$ & $5(4.35)$ & & & & & $\begin{array}{l}225 \\
(97.83)\end{array}$ & $\begin{array}{l}5 \\
(2.17)\end{array}$ & & & & \\
\hline
\end{tabular}

$p_{*}:$ NAFLD group vs. control group; $p_{2}$ : CAD group vs. control group; $p_{3}:$ NAFLD + CAD group vs. control group; $p_{4}:$ NAFLD + CAD group vs. NAFLD group.

SPSS 23.0 was used for testing and $p<0.05$ was considered statistically significant.

Abbreviations: CAD, coronary artery disease; NAFLD, nonalcoholic fatty liver disease.

Table 4. Association between genotype and the risk of NAFLD, CAD, and CAD \& NAFLD*

\begin{tabular}{lllllllll}
\hline & OR $(95 \% \mathrm{CI})$ & $p_{1}$ & OR $(95 \% \mathrm{CI})$ & $p_{2}$ & OR $(95 \% \mathrm{CI})$ & $p_{3}$ & OR $(95 \% \mathrm{CI})$ & $p_{4}$ \\
\hline $\mathrm{CC}$ & 1 & 0.93 & 1 & 0.29 & 1 & 0.07 & 1 & 0.09 \\
$\mathrm{CT}+\mathrm{TT}$ & $0.97(0.48-1.96)$ & & $1.42(0.75-2.67)$ & & $0.39(0.15-1.07)$ & & $0.41(0.14-1.16)$ \\
\hline
\end{tabular}

$p_{*}$ : NAFLD group vs. control group; $p_{2}$ : CAD group vs. control group; $p_{3}$ : NAFLD \& CAD group vs. control group; $p_{4}:$ NAFLD + CAD group vs. NAFLD group.

*Binary logistic analysis was used for testing.

Abbreviations: CAD, coronary artery disease; CI, confidence interval; NAFLD, nonalcoholic fatty liver disease; OR, odds ratio.

Table 5. Distribution of genotype frequency and allele frequency of TCF7L2 rs7903146 in obese and non-obese subjects*

\begin{tabular}{|c|c|c|c|c|}
\hline & NAFLD group, $n(\%)$ & Non-NAFLD group, $n(\%)$ & $x_{2}$ & $p$ \\
\hline \multicolumn{5}{|l|}{ Obese } \\
\hline \multicolumn{5}{|l|}{ Genotype } \\
\hline $\mathrm{CC}$ & $151(91.5)$ & 149 (90.9) & \multirow[t]{3}{*}{0.045} & \multirow[t]{3}{*}{0.832} \\
\hline $\mathrm{CT}+\mathrm{TT}$ & $14(8.5)$ & $15(9.1)$ & & \\
\hline \multicolumn{3}{|l|}{ Allele } & & \\
\hline $\mathrm{C}$ & $316(95.76)$ & $313(95.43)$ & \multirow[t]{2}{*}{0.043} & \multirow[t]{2}{*}{0.836} \\
\hline $\mathrm{T}$ & $14(4.24)$ & $15(4.57)$ & & \\
\hline \multicolumn{5}{|l|}{ Non-obese } \\
\hline \multicolumn{5}{|c|}{ Genotype } \\
\hline $\mathrm{CC}$ & $84(94.38)$ & $175(85.78)$ & \multirow[t]{3}{*}{4.466} & \multirow[t]{2}{*}{0.035} \\
\hline $\mathrm{CT}+\mathrm{TT}$ & $5(5.63)$ & $29(14.22)$ & & \\
\hline \multicolumn{4}{|l|}{ Allele } & \\
\hline $\mathrm{C}$ & $171(96.07)$ & $378(92.65)$ & \multirow[t]{2}{*}{2.451} & \multirow[t]{2}{*}{0.117} \\
\hline $\mathrm{T}$ & $7(3.93)$ & $30(7.35)$ & & \\
\hline
\end{tabular}

* SPSS 23.0 was used for testing.

$p<0.05$ was considered a statistically significant difference.

Abbreviation: NAFLD, nonalcoholic fatty liver disease.

\section{Discussion}

Genetic factors significantly participate in the development and progression of NAFLD and CAD. ${ }^{25,26}$ In this study, we investigated the relationship of TCF7L2 rs7903146 with the risk of developing NAFLD and CAD in a Chinese Han popula- tion. As the results showed, there were no differences in the TCF7L2 rs7903146 genotype and allele distribution between the NAFLD group and the control group, the CAD group and the control group, the NAFLD + CAD group and the control group, and the NAFLD + CAD group and the NAFLD group. In addition, the $C T+\Pi$ genotype did not increase the risk of 
Yan X. et al: Association of TCF7L2 with NAFLD and CAD

Table 6. Association of TCF7L2 rs7903146 genotype and NAFLD in non-obese subjects*

\begin{tabular}{|c|c|c|c|c|c|}
\hline & \multicolumn{2}{|l|}{ Unadjusted } & & \multicolumn{2}{|l|}{ Adjusted* } \\
\hline & OR $(95 \% \mathrm{CI})$ & $P$ & & OR $(95 \% \mathrm{CI})$ & $P$ \\
\hline $\mathrm{CC}$ & 1 & \multirow[t]{2}{*}{0.041} & $\mathrm{CC}$ & 1 & \multirow[t]{2}{*}{0.025} \\
\hline $\mathrm{CT}+\mathrm{TT}$ & $0.359(0.134-0.961)$ & & $\mathrm{CT}+\mathrm{TT}$ & $0.245(0.072-0.837)$ & \\
\hline
\end{tabular}

* Binary logistic regression model was adjusted for age, gender, TC, TG and LDL.

Abbreviations: CI, confidence interval; LDL, low-density lipoprotein; NAFLD, nonalcoholic fatty liver disease; OR, odds ratio; TC, total cholesterol; TG, triglyceride.

developing NAFLD, CAD, and NALFD + CAD in the general population, and did not increase the risk of developing CAD in the patients with NAFLD. However, the TCF7L2 rs7903146 $\mathrm{CT}+\mathrm{TT}$ genotype was a protective factor for NAFLD in the non-obese subjects.

As a component of the Wnt/beta-catenin signaling pathway, TCF7L2 participates in a variety of physiological pathways, such as cell proliferation, differentiation, apoptosis, and oxidative stress, all the effect of TCF7L2 which can be exerted throughout growth, zoning, heterogenesis, and other intrinsic metabolism processes of liver. ${ }^{27-29}$ The relationship of TCF7L2 with metabolism was first found by Grant et al., ${ }^{30}$ who found that the microsatellite of TCF7L2 intron 3, DG10S478, was associated with type 2 diabetes in an Icelandic population. The gene's expression product, a high-mobility family box, contains transcription factors that influence blood glucose homeostasis. Subsequent studies revealed several potential functions of TCF7L2, including decreasing insulin secretion, impairing incretin effect, and increasing insulin resistance. ${ }^{31-33}$ In addition, TCF7L2 rs7903146 has been reported to be relevant to lipid disorders, NAFLD, obesity and hypertension. ${ }^{14,18,34}$ Corella et al. ${ }^{20}$ reported the correlation between TCF7L2 rs7903146 and multiple lipid indexes, but the potential molecular mechanism by which TCF7L2 rs7903146 induces abnormal hepatic metabolism remains unknown.

Some researchers reported that TCF7L2 rs7930146 can regulate open chromatin and genetic transcription in pancreas, thus increasing the risk of diabetes. ${ }^{31,35}$ In addition, Dorota et $a .^{36}$ found that the expression of TCF7L2 in liver may be regulated by weight loss. However, the evidence of how TCF7L2 rs7903146 participates in hepatic lipid metabolism remains unclear. Musso et al. ${ }^{14}$ found that the TCF7L2 rs7903146 CT/TT genotype was a risk factor for NAFLD. In that study, the results indicated that the TCF7L2 rs7903146 polymorphism did not associate with the risk of developing NAFLD, CAD, or NAFLD + CAD in the general population, and did not associate with the risk of developing NAFLD + CAD. Except for BMI in the control group, the differences of clinical parameters between the TCF7L2 rs7903146 T allele carriers and non-carriers in each group were also not significant. Further analysis found that the distribution of the CT + $\Pi$ genotype and the CC genotype was significantly different between the NAFLD patients and non-NAFLD patients in the non-obese group. After adjustment for gender and age, and serum lipids, the CT + TT genotype was still a protective factor for the development of NAFLD in the non-obese subjects. Previous study determined that the minor allele frequency of the rs7903146 Tallele varied according to the difference of region (the minor allele frequencies in Vietnam, Estonia, Sweden, Caucasia, India, East Asia were 4.63, 19.82, 22.5, 29, 28.1, and 3.2 , respectively). ${ }^{37}$ In this study, the minor allele fre- quency of rs7903146 was 4.63, which was similar to the minor allele frequency of East Asia. Development of NAFLD was affected by ethnic, genetic, dietary, and environmental factors; therefore, the difference of the effect of TCF7L2 rs7903146 polymorphism on the risk of developing NAFLD in Chinese and other countries may be affected by ethnic and genetic factors.

There were several limitations in this study. First, all the NAFLD patients were diagnosed by ultrasound rather than the liver biopsy; therefore, the diagnosis of NAFLD may not be very accurate. Second, the sample size was relatively small, which may affect the conclusion. Third, our conclusion cannot reflect the association between TCF7L2 rs7903146 polymorphism and the risk of developing NAFLD in other countries, due to all the subjects in this study being of the Chinese Han population.

\section{Conclusions}

This study investigated the relationship of TCF7L2 rs7903146 polymorphism with the risk of NAFLD, CAD, and NAFLD + CAD in a Chinese Han population, for the first time. The results suggest that TCF7L2 rs7903146 was not associated with the risk of developing NAFLD, CAD, and NAFLD + CAD in our Chinese Han population, and TCF7L2 rs7903146 did not affect the serum lipid metabolism. In the non-obese subpopulation, the TCF7L2 rs7903146 CT + TT genotype was a protective factor for the development of NAFLD. More subjects of other ethnicity should be included to further investigate the association of TCF7L2 rs7903146 with the risk of NAFLD, and the potential mechanism underlying the TCF7L2 rs7903146 variant's affect the lipid metabolism should be illustrated.

\section{Funding}

This study was supported by a grant from the National Natural Science Foundation of China (31770837).

\section{Conflict of interest}

The authors have no conflict of interests related to this publication.

\section{Author contributions}

Study concept and design ( $Y X$ and $S L$ ), acquisition of the data $(\mathrm{XY}, \mathrm{WJ}, \mathrm{JZ}$, and $\mathrm{MW})$, analysis and interpretation of the data ( $Y X$ and $W J)$, drafting of the manuscript: (XY), critical revision of the manuscript for important intellectual content ( $Y X$ and LS), supervision (YX). All the authors read and approved the final manuscript. 


\section{References}

[1] Li J, Zou B, Yeo YH, Feng Y, Xie X, Lee DH, et al. Prevalence, incidence, and outcome of non-alcoholic fatty liver disease in Asia, 1999-2019: a systematic review and meta-analysis. Lancet Gastroenterol Hepatol 2019;4:389-398. doi: 10.1016/S2468-1253(19)30039-1.

[2] Nseir W, Shalata A, Marmor A, Assy N. Mechanisms linking nonalcoholic fatty liver disease with coronary artery disease. Dig Dis Sci 2011;56:3439-3449. doi: 10.1007/s10620-011-1767-y.

[3] Vanwagner LB, Bhave M, Te HS, Feinglass J, Alvarez L, Rinella ME. Patients transplanted for nonalcoholic steatohepatitis are at increased risk for postoperative cardiovascular events. Hepatology 2012;56:1741-1750. doi: 10. 1002/hep.25855.

[4] Assy N, Djibre A, Farah R, Grosovski M, Marmor A. Presence of coronary plaques in patients with nonalcoholic fatty liver disease. Radiology 2010; 254:393-400. doi: 10.1148/radiol.09090769.

[5] Li XL, Sui JQ, Lu LL, Zhang NN, Xu X, Dong QY, et al. Gene polymorphisms associated with non-alcoholic fatty liver disease and coronary artery disease: a concise review. Lipids Health Dis 2016;15:53. doi: 10.1186/s12944-0160221-8.

[6] Wu S, Wu F, Ding $Y$, Hou J, Bi J, Zhang Z. Association of non-alcoholic fatty liver disease with major adverse cardiovascular events: A systematic review and meta-analysis. Sci Rep 2016;6:33386. doi: 10.1038/srep33386.

[7] Akabame S, Hamaguchi M, Tomiyasu K, Tanaka M, Kobayashi-Takenaka $Y$ Nakano $\mathrm{K}$, et al. Evaluation of vulnerable coronary plaques and non-alcoholic fatty liver disease (NAFLD) by 64-detector multislice computed tomography (MSCT). Circ J 2008;72:618-625. doi: 10.1253/circj.72.618.

[8] Yilmaz Y, Kurt R, Yonal O, Polat N, Celikel CA, Gurdal A, et al. Coronary flow reserve is impaired in patients with nonalcoholic fatty liver disease: association with liver fibrosis. Atherosclerosis $2010 ; 211: 182-186$. doi: $10.1016 / \mathrm{j}$. atherosclerosis.2010.01.049.

[9] Shitashige $M$, Hirohashi $S$, Yamada T. Wnt signaling inside the nucleus. Cancer Sci 2008;99:631-637. doi: 10.1111/j.1349-7006.2007.00716.x.

[10] Grove EA. Wnt signaling meets internal dissent. Genes Dev 2011;25:17591762. doi: $10.1101 /$ gad.17594311.

[11] Waterman ML. Lymphoid enhancer factor/T cell factor expression in colorectal cancer. Cancer Metastasis Rev 2004;23:41-52. doi: 10.1023/a: 1025858928620.

[12] Ye F, Chen Y, Hoang T, Montgomery RL, Zhao XH, Bu H, et al. HDAC1 and HDAC2 regulate oligodendrocyte differentiation by disrupting the betacatenin-TCF interaction. Nat Neurosci 2009;12:829-838. doi: 10.1038/nn. 2333.

[13] Batlle E, Henderson JT, Beghtel H, van den Born MM, Sancho E, Huls G, et al. Beta-catenin and TCF mediate cell positioning in the intestinal epithelium by controlling the expression of EphB/ephrinB. Cell 2002;111:251-263. doi: 10.1016/s0092-8674(02)01015-2.

[14] Musso G, Gambino R, Pacini G, Pagano G, Durazzo M, Cassader M. Transcription factor 7-like 2 polymorphism modulates glucose and lipid homeostasis, adipokine profile, and hepatocyte apoptosis in NASH. Hepatology 2009;49: 426-435. doi: 10.1002/hep.22659.

[15] Musso G, Gambino R, Pacini G, De Michieli F, Cassader M. Prolonged saturated fat-induced, glucose-dependent insulinotropic polypeptide elevation is associated with adipokine imbalance and liver injury in nonalcoholic steatohepatitis: dysregulated enteroadipocyte axis as a novel feature of fatty liver. Am J Clin Nutr 2009;89:558-567. doi: 10.3945/ajcn.2008.26720.

[16] Kim SJ, Nian C, McIntosh CH. Resistin is a key mediator of glucose-dependent insulinotropic polypeptide (GIP) stimulation of lipoprotein lipase (LPL) activity in adipocytes. J Biol Chem 2007;282:34139-34147. doi: 10. 1074/jbc.M704896200.

[17] Hansotia T, Maida A, Flock G, Yamada Y, Tsukiyama K, Seino Y, et al. Extrapancreatic incretin receptors modulate glucose homeostasis, body weight, and energy expenditure. J Clin Invest 2007;117:143-152. doi: 10. $1172 / \mathrm{JCI} 25483$

[18] Huertas-Vazquez A, Plaisier C, Weissglas-Volkov D, Sinsheimer ], CanizalesQuinteros S, Cruz-Bautista I, et al. TCF7L2 is associated with high serum triacylglycerol and differentially expressed in adipose tissue in families with familial combined hyperlipidaemia. Diabetologia 2008;51:62-69. doi: 10. 1007/s00125-007-0850-6.

[19] Chen X, Ayala I, Shannon C, Fourcaudot M, Acharya NK, Jenkinson CP, et al. The diabetes gene and wnt pathway effector TCF7L2 regulates adipocyte development and function. Diabetes 2018;67:554-568. doi: 10 . 2337/db17-0318.

[20] Corella D, Carrasco P, Sorlí JV, Estruch R, Rico-Sanz J, Martínez-González MÁ et al. Mediterranean diet reduces the adverse effect of the TCF7L2rs7903146 polymorphism on cardiovascular risk factors and stroke incidence: a randomized controlled trial in a high-cardiovascular-risk population. Diabetes Care 2013;36:3803-3811. doi: 10.2337/dc13-0955.

[21] Oktavianthi S, Saraswati MR, Suastika K, Dwipayana P, Sulfianti A, Hayati RF, et al. Transcription factor 7-like 2 single nucleotide polymorphisms are associated with lipid profile in the Balinese. Mol Biol Rep 2018;45:1135-1143. doi: $10.1007 / \mathrm{s} 11033-018-4265-x$

[22] Pineda-Tenor D, Berenguer J, Jiménez-Sousa MA, Carrero A, García-Álvarez M, Aldámiz-Echevarria T, et al. rs7903146 polymorphism at transcription factor 7 like 2 gene is associated with total cholesterol and lipoprotein profile in HIV/hepatitis C virus-coinfected patients. AIDS Res Hum Retroviruses 2015;31:326-334. doi: 10.1089/aid.2014.0195.

[23] Perez-Martinez P, Perez-Caballero AI, Garcia-Rios A, Yubero-Serrano EM, Camargo A, Gomez-Luna MJ, et al. Effects of rs7903146 variation in the Tcf7I2 gene in the lipid metabolism of three different populations. PLoS One 2012;7:e43390. doi: 10.1371/journal.pone.0043390.

[24] Human experimentation: Code of ethics of the World Medical Association (Declaration of Helsinki). Can Med Assoc J. 1964;91:619.

[25] Younossi Z, Anstee QM, Marietti M, Hardy T, Henry L, Eslam M, et al. Global burden of NAFLD and NASH: trends, predictions, risk factors and prevention. Nat Rev Gastroenterol Hepatol 2018;15:11-20. doi: 10.1038/nrgastro. 2017.109.

[26] Khera AV, Kathiresan S. Genetics of coronary artery disease: discovery, biology and clinical translation. Nat Rev Genet 2017;18:331-344. doi: 10. 1038/nrg.2016.160.

[27] Monga SP. Role of Wnt/ $\beta$-catenin signaling in liver metabolism and cancer. Int J Biochem Cell Biol. 2011;43:1021-1029. doi: 10.1016/j.biocel.2009.09. 001.

[28] Jungermann K, Katz N. Functional specialization of different hepatocyte populations. Physiol Rev 1989;69:708-764. doi: 10.1152/physrev.1989.69.3. 708.

[29] Gebhardt R, Hovhannisyan A. Organ patterning in the adult stage: the role of Wnt/beta-catenin signaling in liver zonation and beyond. Dev Dyn 2010; 239:45-55. doi: 10.1002/dvdy.22041.

[30] Grant SF, Thorleifsson G, Reynisdottir I, Benediktsson R, Manolescu A, Sainz J, et al. Variant of transcription factor 7-like 2 (TCF7L2) gene confers risk of type 2 diabetes. Nat Genet 2006;38:320-323. doi: 10.1038/ng1732.

[31] Lyssenko V, Lupi R, Marchetti P, Del Guerra S, Orho-Melander M, Almgren $P_{\text {, }}$ et al. Mechanisms by which common variants in the TCF7L2 gene increase risk of type 2 diabetes. J Clin Invest 2007;117:2155-2163. doi: 10 1172/JCI30706.

[32] Florez JC, Jablonski KA, Bayley N, Pollin TI, de Bakker PI, Shuldiner AR, et al. TCF7L2 polymorphisms and progression to diabetes in the Diabetes Prevention Program. N Engl J Med 2006;355:241-250. doi: 10 1056/NEJMoa062418.

[33] Damcott CM, Pollin TI, Reinhart LJ, Ott SH, Shen H, Silver KD, et al. Polymorphisms in the transcription factor 7-like 2 (TCF7L2) gene are associated with type 2 diabetes in the Amish: replication and evidence for a role in both insulin secretion and insulin resistance. Diabetes 2006;55:2654-2659. doi: $10.2337 / \mathrm{db} 06-0338$.

[34] Samaan Z, Lee YK, Gerstein HC, Engert JC, Bosch J, Mohan V, et al. Obesity genes and risk of major depressive disorder in a multiethnic population: a cross-sectional study. J Clin Psychiatry 2015;76:e1611-e1618. doi: 10. 4088/JCP.14m09720.

[35] Gaulton KJ, Nammo T, Pasquali L, Simon JM, Giresi PG, Fogarty MP, et al. A map of open chromatin in human pancreatic islets. Nat Genet 2010;42:255259. doi: $10.1038 / \mathrm{ng} .530$.

[36] Kaminska D, Kuulasmaa T, Venesmaa S, Käkelä P, Vaittinen M, Pulkkinen L, et al. Adipose tissue TCF7L2 splicing is regulated by weight loss and associates with glucose and fatty acid metabolism. Diabetes 2012;61:28072813. doi: $10.2337 / \mathrm{db} 12-0239$.

[37] Tong $Y$, Lin $Y$, Zhang $Y$, Yang J, Zhang $Y$, Liu H, et al. Association between TCF7L2 gene polymorphisms and susceptibility to type 2 diabetes mellitus: large Human Genome Epidemiology (HuGE) review and meta-analysis. BMC Med Genet 2009;10:15. doi: 10.1186/1471-2350-10-15 